\title{
A Permutation Test for Unit Root in an Autoregressive Model
}

\author{
Jiexiang Li ${ }^{1}$, Lanh Tran ${ }^{2}$, Sa-aat Niwitpong ${ }^{3}$ \\ ${ }^{1}$ Department of Mathematics, College of Charleston, Charleston, USA \\ ${ }^{2}$ Department of Statistics, Indiana University, Bloomington, USA \\ ${ }^{3}$ Department of Applied Statistics, King Mongkut's University of Technology, Bangkok, Thailand \\ Email: 1ij@cofc.edu,tran@indiana.edu,snw@kmutnb.ac.th
}

Received September 23, 2013; revised October 23, 2013; accepted October 30, 2013

Copyright (C) 2013 Jiexiang Li et al. This is an open access article distributed under the Creative Commons Attribution License, which permits unrestricted use, distribution, and reproduction in any medium, provided the original work is properly cited.

\begin{abstract}
A permutation test (based on a finite random sample of permutations) for unit root in an autoregressive process is considered. The test can easily be carried out in practice and the proposed permutation test is neither limited to large sample sizes nor normal white noises. Simulations show that the power of the permutation test is reasonable when sample sizes are small or when the white noises have a heavy tailed distribution. The test is shown to be consistent.
\end{abstract}

Keywords: Permutation Test; Autoregressive; Nonstationary

\section{Introduction}

Let $Y_{1}, Y_{2}, \cdots, Y_{n+1}$ be $n+1$ observations of the real valued autoregressive model

$$
Y_{t}=a Y_{t-1}+e_{t}
$$

for $0<a \leq 1$ and $e_{t}$ is a sequence of independent identically distributed random variables with mean zero and variance $\sigma^{2}$ and $Y_{1}=0$. Tests of

$$
H_{0}: a=1 \text { versus } H_{A}: 0<a<1 \text {, }
$$

are often referred to as tests for unit root. The hypothesis that $a=1$ is of interest in applications because it corresponds to the hypothesis that it is appropriate to transform the times series by difference. In [1] and [2], the authors derived the limit distribution of the statistic $n(\hat{a}-1)$ with

$$
\hat{a}=\left(\sum_{i=2}^{n+1} Y_{t-1}^{2}\right)^{-1} \sum_{t=2}^{n+1} Y_{t} Y_{t-1}
$$

under the unit root assumption $a=1$. However, [1] and [2] are limited to large sample sizes or normal white noises. When sample sizes are small and white noises are from distributions with heavy tails, to test for the presence of unit root, we can use the permutation test proposed in the paper.

Under $H_{0}, Y_{t}$ is not stationary and the variance of $Y_{t}$ is $t \sigma^{2}$. When $H_{0}$ is true, $Y_{t}$ is sometimes called a random walk and $X_{t}=Y_{t+1}-Y_{t}, \quad t=1,2, \cdots, n$ are independent identically distributed r.v.'s. In Economics, it is important to characterize the velocity, or stock price as a random walk. Another way of phrasing $H_{0}$ is that whatever determinants of velocity and their individual stochastic structure may be, their combined effect is such that successive changes in velocity are essentially independent. This would imply that of the past history available at any given date only the current observation is relevant for prediction. Testing for unit root is equivalent to testing for serial independence in sequence $X_{1}, \cdots, X_{n}$. For some literature on tests for serial dependence, see [3] and [4] and the references therein. We define

$$
T_{n}=\sum_{i=1}^{n-1} X_{i} X_{i+1},
$$

and for each random permutation of the vector $\left(X_{1}, \cdots, X_{n}\right)$, say, $\left(X_{1 \ell}, \cdots, X_{n \ell}\right)$, denote

$$
T_{n \ell}=\sum_{i=1}^{n-1} X_{i \ell} X_{(i+1) \ell} .
$$

If $H_{0}$ holds then $\left(X_{1}, \cdots, X_{n}\right)=\left(e_{2}, \cdots, e_{n+1}\right)$. The distribution of a random vector of i.i.d. random variables is invariant under any permutation of its coordinates. Thus $T_{n}$ and $T_{n \ell}$ have the same distribution if $H_{0}$ is true. Based on the invariance of the distribution of the 
statistic $T_{n}$ under permutations when $H_{0}$ holds, we propose a permutation test for unit root using $T_{n}$ as our pivot test statistic. This test is easy to perform with a computer and the test makes little assumptions on the probability distribution of the white noise and also it works for small samples. Construction of this test will be presented in Section 2. The consistency of our test is shown in Section 3. A simulation study of our test is provided in Section 4. Time series of velocity of money observed between 1869 and 1960 is investigated in Section 5 .

\section{Steps Used in Our Permutation Test}

We assume that the white noise $e_{t}$ is a sequence of independent identically distributed random variables with mean zero and variance $\sigma^{2}$. In addition, $E\left(e_{t}^{4}\right)<\infty$.

We will often write $T_{n}$ simply as $T$ for brevity. Note that $T$ is more likely to be negative under $H_{A}$ (see Lemma 3.2 below). To summarize, the permutation test is carried out as follows.

1) Set a predetermined level $\alpha$. Permute the $n$ observations $\left\{X_{1}, X_{2}, \cdots, X_{n}\right\}$. There are a total of $n$ ! permutations. For each permutation, compute the $T$ statistic. Under $H_{0}$, the $T$ statistics have the same probability distribution for all of the $n$ ! permutations. The $T$ statistic computed from the observations (not permuted) is referred to as $T_{o b s}$.

2) Compute the $p$-value as the proportion of $T$ 's less than or equal to $T_{o b s}$, that is,

$$
p \text {-value }=\frac{\text { number of } T^{\prime} s \leq T_{o b s}}{n !} .
$$

Conclude that the test is statistically significant if the computed $p$-value is less than or equal to $\alpha$.

This test is limited by prohibitive calculation and hard to carry out if $n$ is a large number. Instead of using all $n$ ! permutations to compute the $p$-value, we obtain a random sample $R$ of permutations and then carry out the test as follows:

1) Set a predetermined level $\alpha$. Compute the $T$ statistic for each of the $R$ sampled permutation.

2) Compute the $p$-value as the proportion of $T$ 's less than or equal to $T_{o b s}$, that is,

$$
p \text {-value }=\frac{\text { number of } T^{\prime} s \leq T_{o b s}}{R} .
$$

Conclude that the test is statistically significant if the computed $p$-value is less than or equal to $\alpha$.

The approximate $p$-value is now equal to the fraction of $T$ 's that are less than or equal to $T_{o b s}$. The theory of the binomial distribution tells us that the approximate value has about a $95 \%$ chance of being within

$$
\pm 2 \sqrt{p(1-p) / R}
$$

of the true $p$-value. We will denote the permutation test based on a random sample of $R$ permutations by $\phi_{R}$.

Remark. Consider the lag one autocorrelation

$$
r_{1}=\frac{\sum_{i=1}^{n-1}\left(X_{i}-\bar{X}\right)\left(X_{i+1}-\bar{X}\right)}{\sum_{i=1}^{n}\left(X_{i}-\bar{X}\right)^{2}} .
$$

Using $T$ is "almost" equivalent to using $r_{1}$ to perform our test. We leave it to the reader to verify this.

\section{Consistency of $\phi_{R}$}

Consistency of hypothesis tests is a desirable property. In this section, we will show that the permutation test based on random sampling $R$ permutations is consistent, that is, the probability of correctly rejecting the null hypothesis $H_{0}$ tends to 1 as the sample size goes to infinity when $0<a<1$. In other words,

Theorem 3.1 Suppose $H_{a}$ is an arbitrary simple hypothesis that the autoregressive parameter $a$ is between 0 and 1 , that is $H_{a} \in H_{A}$. Then

$$
P_{H_{a}}\left[\text { Reject } H_{0}\right] \rightarrow 1
$$

as $n \rightarrow \infty$

Lemma 3.1 Under $H_{A}$, for any integer $m \geq 1$,

$$
E\left(X_{1} X_{1+m}\right)=\frac{\sigma^{2}}{1-a^{2}}\left(2 a^{m}-a^{m-1}-a^{m+1}\right) \text {. }
$$

Proof. We can write $Y_{t}$ as

$$
Y_{t}=\sum_{u=0}^{\infty} a^{u} e_{t-u} .
$$

Using (1), it is easily seen that under $H_{A}$, the AR(1) process $Y_{t}$ has mean zero with

$$
\operatorname{Var}\left(Y_{t}\right)=\frac{\sigma^{2}}{1-a^{2}}
$$

and

$$
E\left(Y_{t} Y_{t+m}\right)=\frac{\sigma^{2}}{1-a^{2}} a^{m}
$$

Clearly,

$E\left(X_{1} X_{1+m}\right)=E\left(Y_{2} Y_{2+m}-Y_{2} Y_{1+m}-Y_{1} Y_{2+m}+Y_{1} Y_{1+m}\right)$.

In particular,

$$
E\left(X_{1} X_{2}\right)=-\frac{\sigma^{2}(1-a)}{(1+a)},
$$

which is negative under $H_{A}$. Note 


$$
\begin{aligned}
& E\left(Y_{1}^{2} Y_{2}^{2}\right) \\
= & E\left(\sum_{u=0}^{\infty} a^{u} e_{1-u} \sum_{v=0}^{\infty} a^{v} e_{1-v} \sum_{p=0}^{\infty} a^{p} e_{2-p} \sum_{q=0}^{\infty} a^{q} e_{2-q}\right) \\
= & \sum_{u=0}^{\infty} a^{2+4 u} E\left(e_{1-u}^{4}\right)+2 \sum_{u=0}^{\infty} \sum_{v \neq u} a^{1+2 u} a^{1+2 v} \sigma^{4}+\sum_{u=0}^{\infty} \sum_{v \neq 1+u} a^{2 u} a^{2 v} \sigma^{4} \\
= & \frac{a^{2}}{1-a^{4}} E\left(e_{t}^{4}\right)+2\left[\frac{a^{2}}{\left(1-a^{2}\right)^{2}}-\frac{a^{2}}{1-a^{4}}\right] \sigma^{4} \\
& +\left[\frac{1}{\left(1-a^{2}\right)^{2}}-\frac{a^{2}}{1-a^{4}}\right] \sigma^{4} .
\end{aligned}
$$

Lemma 3.2 Under $H_{A}, T_{n} / n$ converges in probability to $E\left(X_{1} X_{2}\right)=-\frac{\sigma^{2}(1-a)}{1+a}$

Proof. Under $H_{A}$, time series $Y_{t}$ is stationary and so is time series $X_{t} X_{t+1}$. Thus

$$
E\left(\frac{T_{n}}{n-1}\right)=E\left(X_{1} X_{2}\right)
$$

it suffices to show that

$$
\operatorname{Var}\left(\frac{T_{n}}{n-1}\right) \rightarrow 0
$$

$$
\begin{aligned}
& \operatorname{Var}\left(T_{n}\right) \\
& =(n-1) \operatorname{Var}\left(X_{1} X_{2}\right)+2 \sum_{1 \leq i<j \leq n-1} \operatorname{Cov}\left(X_{i} X_{i+1}, X_{j} X_{j+1}\right) \\
& =(n-1) \operatorname{Var}\left(X_{1} X_{2}\right)+2 \sum_{j=2}^{n-1}(n-j) \operatorname{Cov}\left(X_{1} X_{2}, X_{j} X_{j+1}\right) \\
& =(n-1) \operatorname{Var}\left(X_{1} X_{2}\right)+2(n-2) \operatorname{Cov}\left(X_{1} X_{2}, X_{2} X_{3}\right) \\
& +2(n-3) \operatorname{Cov}\left(X_{1} X_{2}, X_{3} X_{4}\right) \\
& +2 \sum_{j=4}^{n-1}(n-j)\left[E\left(X_{1} X_{2} X_{j} X_{j+1}\right)-\left(E\left(X_{1} X_{2}\right)\right)^{2}\right] .
\end{aligned}
$$

By (3), it is sufficient to show that

$$
\frac{\sum_{j=4}^{n-1}(n-j)\left[E\left(X_{1} X_{2} X_{j} X_{j+1}\right)-\left(E\left(X_{1} X_{2}\right)\right)^{2}\right]}{(n-1)^{2}} \rightarrow 0
$$

as $n \rightarrow \infty$.

For $j \geq 4$, consider $E\left(X_{1} X_{2} X_{j} X_{j+1}\right)$. Following the proof of (2), we have

$$
\begin{aligned}
& E\left(X_{1} X_{2} X_{j} X_{j+1}\right) \\
& =\frac{-a^{2 j-3}+3 a^{2 j-2}-3 a^{2 j-1}+2 a^{2 j}}{1-a^{4}} E\left(e_{t}^{4}\right) \\
& +\frac{3 a^{2 j+2}-3 a^{2 j+1}-a^{2 j+3}}{1-a^{4}} E\left(e_{t}^{4}\right) \\
& +\frac{1-4 a+6 a^{2}-4 a^{3}+a^{4}}{\left(1-a^{2}\right)^{2}} \sigma^{4} \\
& +\frac{3 a^{2 j-3}-9 a^{2 j-2}+9 a^{2 j-1}-6 a^{2 j}}{1-a^{4}} \sigma^{4} \\
& +\frac{9 a^{2 j+1}-9 a^{2 j+2}+3 a^{2 j+3}}{1-a^{4}} \sigma^{4} \\
& +\frac{2 a^{2 j-4}-8 a^{2 j-3}+12 a^{2 j-2}-8 a^{2 j-1}+2 a^{2 j}}{\left(1-a^{2}\right)^{2}} \sigma^{4} .
\end{aligned}
$$

It is not hard to see that

$$
\begin{aligned}
& \frac{1}{(n-1)^{2}} \sum_{j=4}^{n-1}(n-j)\left(-a^{2 j-3}+3 a^{2 j-2}-3 a^{2 j-1}+2 a^{2 j}\right) \\
& +\frac{1}{(n-1)^{2}} \sum_{j=4}^{n-1}(n-j)\left(-3 a^{2 j+1}+3 a^{2 j+2}-a^{2 j+3}\right) \rightarrow 0
\end{aligned}
$$

and

$$
\begin{aligned}
& \sum_{j=4}^{n-1}(n-j) \frac{1-4 a+6 a^{2}-4 a^{3}+a^{4}}{(n-1)^{2}\left(1-a^{2}\right)^{2}} \\
& +\sum_{j=4}^{n-1}(n-j) \frac{2 a^{2 j-4}-8 a^{2 j-3}+12 a^{2 j-2}-8 a^{2 j-1}+2 a^{2 j}}{(n-1)^{2}\left(1-a^{2}\right)^{2}} \\
& -\sum_{j=4}^{n-1}(n-j) \frac{(1-a)^{2}}{(n-1)^{2}(1+a)^{2}} \rightarrow 0
\end{aligned}
$$

as $n \rightarrow \infty$. This completes the proof.

On the left of Figures $\mathbf{1}$ and 2, it shows the long run behavior of $T_{n} / n$ based on 10,000 simulations from AR(1) model with white noise from normal $(0,1)$ and uniform $(-1,1)$ respectively.

Lemma 3.3 Under $H_{A}, E\left(X_{1 \ell} X_{2 \ell}\right) \rightarrow 0$ as $n \rightarrow \infty$.

Proof. For a random permutation, $\ell \leq R$, of $X_{1}, X_{2}, \cdots X_{n}$.

$$
P\left(X_{1 \ell} X_{2 \ell}=X_{i} X_{j}\right)=\frac{1}{n(n-1)}
$$

for any $1 \leq i \neq j \leq n$.

$$
\begin{aligned}
& E\left(X_{1 \ell} X_{2 \ell}\right)=E\left(E\left[X_{1 \ell} X_{2 \ell} \mid X_{1}, \cdots, X_{n}\right]\right) \\
& =\sum_{1 \leq i<j \leq n} \frac{2}{n(n-1)} E\left(X_{i} X_{j}\right) .
\end{aligned}
$$


For $i<j$, by stationarity and Lemma 3.1,

$$
\begin{aligned}
& E\left(X_{i} X_{j}\right)=E\left(X_{1} X_{j-i+1}\right) \\
& =\frac{\sigma^{2}}{1-a^{2}}\left(2 a^{j-i}-a^{j-i-1}-a^{j-i+1}\right) .
\end{aligned}
$$

From (4) and (5),

$$
E\left(X_{1 \ell} X_{2 \ell}\right)=\frac{2 \sigma^{2}}{1-a^{2}} \frac{n a-(n-1)-a^{n}}{n(n-1)} .
$$

The proof follows from (6).

Lemma 3.4 Under $H_{A}, E\left(X_{1 \ell} X_{2 \ell} X_{3 \ell} X_{4 \ell}\right) \rightarrow 0$ as $n \rightarrow \infty$.

Proof.

$E\left(X_{1 \ell} X_{2 \ell} X_{3 \ell} X_{4 \ell}\right)=\sum_{1 \leq i<j<k<l \leq n} \frac{4 ! E\left(X_{i} X_{j} X_{k} X_{l}\right)}{n(n-1)(n-2)(n-3)}$. (7)

Note

$$
\begin{aligned}
& E\left(X_{i} X_{j} X_{k} X_{l}\right) \\
& =E\left(Y_{i+1} Y_{j+1} Y_{k+1} Y_{l+1}\right)-E\left(Y_{i+1} Y_{j+1} Y_{k+1} Y_{l}\right) \\
& -E\left(Y_{i+1} Y_{j+1} Y_{k} Y_{l+1}\right)+E\left(Y_{i+1} Y_{j+1} Y_{k} Y_{l}\right) \\
& -E\left(Y_{i+1} Y_{j} Y_{k+1} Y_{l+1}\right)+E\left(Y_{i+1} Y_{j} Y_{k+1} Y_{l}\right) \\
& +E\left(Y_{i+1} Y_{j} Y_{k} Y_{l+1}\right)-E\left(Y_{i+1} Y_{j} Y_{k} Y_{l}\right) \\
& -E\left(Y_{i} Y_{j+1} Y_{k+1} Y_{l+1}\right)+E\left(Y_{i} Y_{j+1} Y_{k+1} Y_{l}\right) \\
& +E\left(Y_{i} Y_{j+1} Y_{k} Y_{l+1}\right)-E\left(Y_{i} Y_{j+1} Y_{k} Y_{l}\right) \\
& +E\left(Y_{i} Y_{j} Y_{k+1} Y_{l+1}\right)-E\left(Y_{i} Y_{j} Y_{k+1} Y_{l}\right) \\
& -E\left(Y_{i} Y_{j} Y_{k} Y_{l+1}\right)+E\left(Y_{i} Y_{j} Y_{k} Y_{l}\right) .
\end{aligned}
$$

After lengthy calculation of $E\left(X_{i} X_{j} X_{k} X_{l}\right)$, we know that to show (7) converges to zero when $n$ goes to infinity, it is sufficient to show

$$
\begin{aligned}
& \quad \sum_{1 \leq i<j<k<l \leq n} \frac{2 a^{j+k+1}-3 a^{j+l+k-1}+3 a^{j+l+k-2}-a^{j+l+k-3}}{n^{4} a^{3 i}} \\
& +\sum_{1 \leq i<j<k<l \leq n} \frac{-a^{j+l+k+3}+3 a^{j+l+k+2}-3 a^{j+l+k+1}}{n^{4} a^{3 i}} \rightarrow 0 ; \\
& \quad \sum_{1 \leq i<j<k<l \leq n} \frac{6 a^{j+l-k-i}-4 a^{j+l-k-i-1}-4 a^{j+l-k-i+1}}{n^{4}} \\
& +\sum_{1 \leq i<j<k<l \leq n} \frac{a^{j+l-k-i-2}+a^{j+l-k-i+2}}{n^{4}} \rightarrow 0 ; \\
& \quad \sum_{1 \leq i<j<k<l \leq n} \frac{12 a^{k+l-j-i}-8 a^{k+l-j-i-1}-8 a^{k+l-j-i+1}}{n^{4}} \\
& \quad+\sum_{1 \leq i<j<k<l \leq n} \frac{2 a^{k+l-j-i-2}+2 a^{k+l-j-i+2}}{n^{4}} \rightarrow 0,
\end{aligned}
$$

which are easy to see.

Lemma 3.5 Under $H_{A}, T_{n \ell} / n$ converges to 0 in probability for all $1 \leq \ell \leq R$.

The proof follows from (3), Lemma 3.3 and 3.4.

On the right of Figures 1 and 2, it shows the long run behavior of $T_{n \ell} / n$ based on 10,000 simulations from AR(1) model with white noise from normal $(0,1)$ and uniform $(-1,1)$ respectively.

Proof of Theorem 3.1. Clearly, under $H_{A}$,

$$
\begin{aligned}
& P\left[T_{n \ell} \geq T_{n}, \text { for all } 1 \leq \ell \leq R\right] \\
& =P\left[T_{n \ell} / n \geq T_{n} / n, \text { for all } 1 \leq \ell \leq R\right],
\end{aligned}
$$

$\operatorname{AR}(1)$ with norm $(0,1)$ noise

$\operatorname{AR}(1)$ with norm $(0,1)$ noise
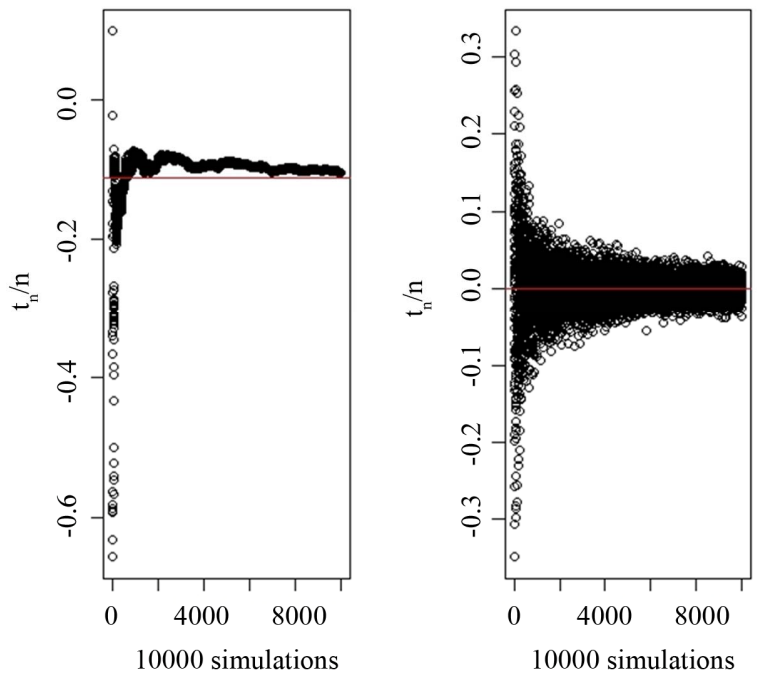

Figure 1. Illustrations of Lemma 3.2 and Lemma 3.5 with normal $(0,1)$ noise and $a=0.8$.

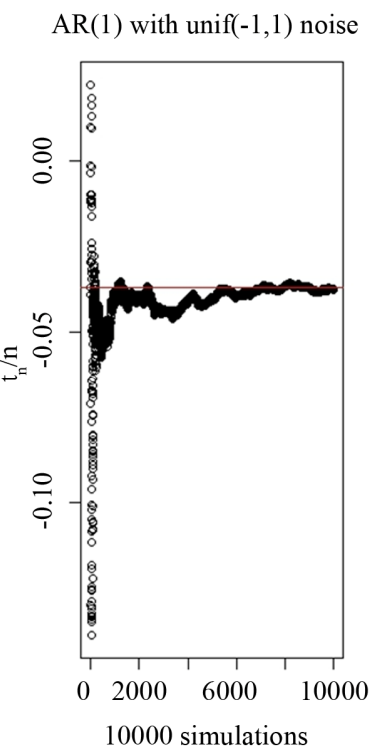

AR(1) with unif(-1,1) noise

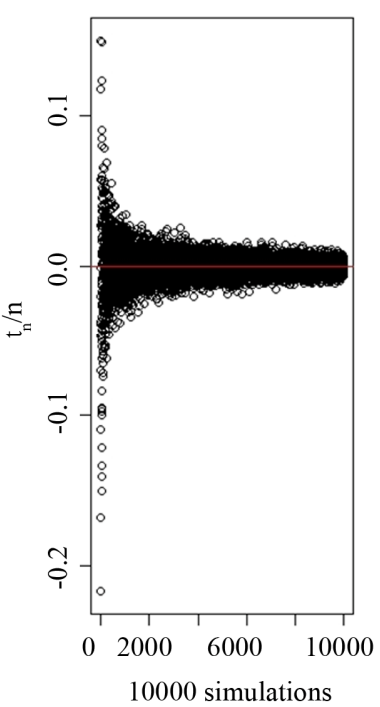

Figure 2. Illustrations of Lemma 3.2 and Lemma 3.5 with uniform $(-1,1)$ noise and $a=0.8$ 
which tends to 1 by Lemma 3.2 and Lemma 3.5. In particular, the probability of rejecting $H_{0}$ under $H_{A}$ tends to 1 .

\section{Simulation Study}

Consider the model $Y_{t}=a Y_{t-1}+e_{t}, \quad t=2, \cdots, n+1$, $Y_{1}=0$ where the $e_{t}$ has contaminated normal distribution. Note the contaminated normal observations were generated in the following way: $70 \%$ of the time an observation is generated from a standard normal distribution while $30 \%$ of the time it is generated from a normal distribution with mean 0 and standard deviation 25. One thousand samples of size $n=5,25,50,100$, 250, 500 were generated for $a=0.2,0.4,0.6,0.8,1$. Permutation tests based on all permutations when samples are small or randomly selected 1000 permutations for unit root were applied to each sample at the significance level 0.05 . The power of the test is tabulated in Table 1 based on 1000 simulated tests for $n=5,25,50$, $100,250,500$ and $a=0.2,0.4,0.6,0.8$. From the table, it is easy to see that the power gets closer to 1 when the sample size increases and this demonstrates the consistency of the proposed permutation test.

\section{An Example}

Reference [5] studied the stochastic structure of velocity in order to determine whether there is a statistical basis for extrapolative prediction. Noting that the velocity of money is defined as the ratio of national income to the stock of money. In the paper they conclude that the logs of the velocity series constructed in [6] are well characterized as a simple random walk. As preliminary analysis, we look at the time series plot of $Y_{t}$, the centered logs of velocity. The pattern in the time series plot is typical of a nonstationary series of the sort which displays no affinity for a mean value. We also note that the autocorrelations of centered logs of velocity series are very large and decline slowly with increasing lag (Figure 3). Now let us look at the time series plot of $X_{t}$, the first differences of centered logs of velocity, and autocorrelations of $X_{t}$ (Figure 4). Judging from the time series plot and autocorrelations of time series $X_{t}$,

Table 1. Power of our permutation test.

\begin{tabular}{ccccccc}
\hline$n$ & 5 & 25 & 50 & 100 & 250 & 500 \\
\hline$a=0.2$ & 0.081 & 0.639 & 0.905 & 0.997 & 1 & 1 \\
$a=0.4$ & 0.051 & 0.398 & 0.71 & 00.933 & 1 & 1 \\
$a=0.6$ & 0.047 & 0.19 & 0.342 & 0.652 & 0.954 & 0.998 \\
$a=0.8$ & 0.045 & 0.09 & 0.147 & 0.196 & 0.449 & 0.706 \\
$a=1$ & 0.054 & 0.046 & 0.047 & 0.044 & 0.048 & 0.055 \\
\hline
\end{tabular}
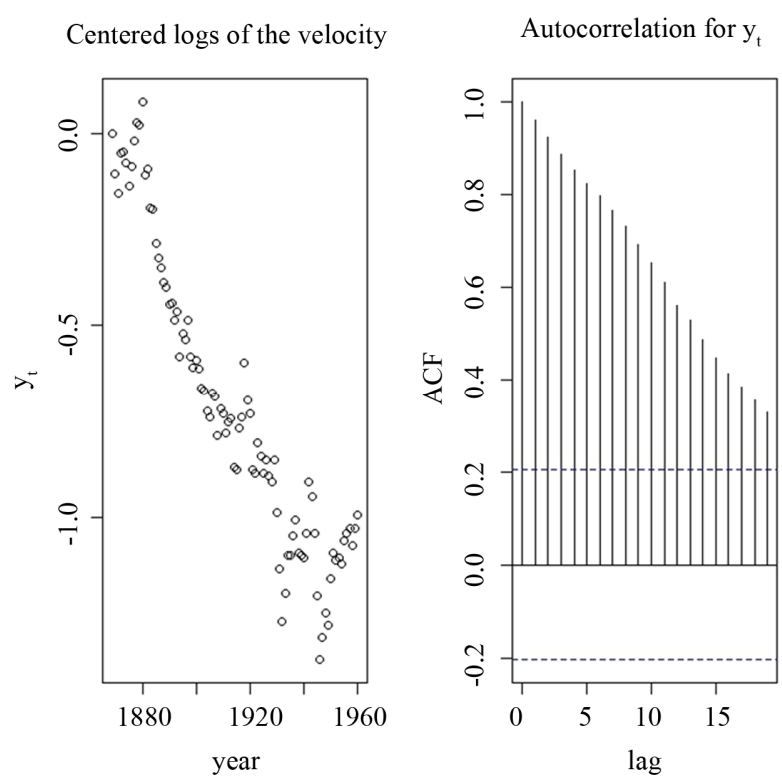

Figure 3. Time series $Y_{t}$ and its autocorrelations.
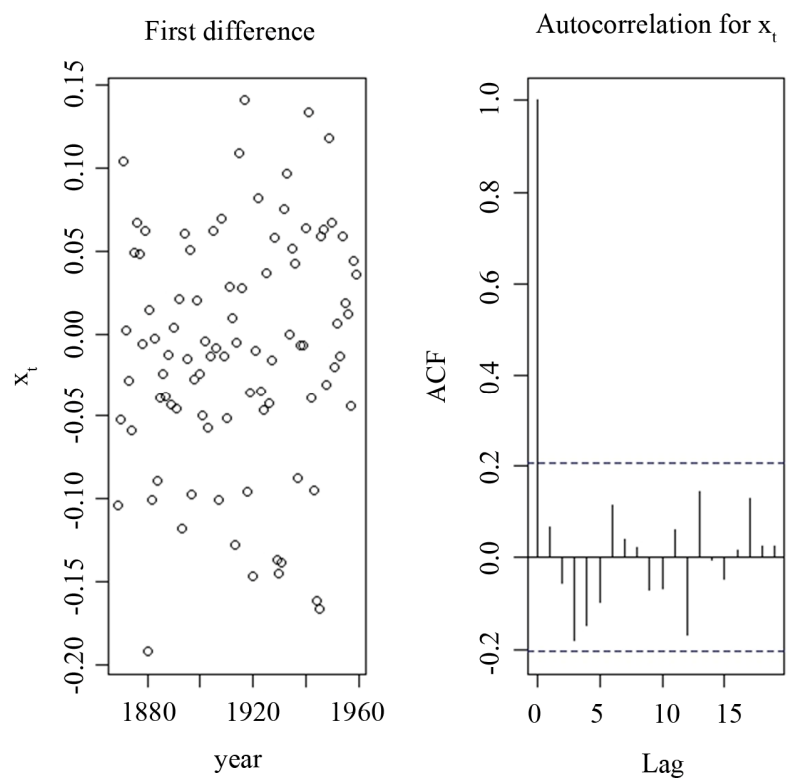

Figure 4. Time series $X_{t}$ and its autocorrelations.

there seems no significant dependence in the time series $X_{t}$. Moving on to the formal analysis, we can see that it is reasonable to fit model $Y_{t}=a Y_{t-1}+e_{t}$ to the centered logs. With an application of permutation test on the centered logs, we obtain the test statistic $T_{o b s}=0.04$, and the $p$-value $=0.7674$. The null hypothesis is not rejected at any reasonable level.

\section{REFERENCES}

[1] W. A. Fuller, "Introduction to Statistical Times Series," John Wiley \& Sons, New York, 1976. 
[2] D. A. Dickey, "Estimation and Hypothesis Testing in Nonstationary Time Series," Ph.D. Dissertation, Iowa State University, Ames, 1976.

[3] N. H. Chan and L. T. Tran, "Nonparametric Tests for Serial Dependence," Journal of Time Series Analysis, Vol. 13, No. 1, 1992, pp. 19-28.

http://dx.doi.org/10.1111/j.1467-9892.1992.tb00092.x

[4] H. J. Skaug and D. Dad Tjóstheim, "A Nonparametric Test of Serial Independence Based on the Empirical Dis- tribution Function. Consistent Nonparametric Multiple Regression: The Fixed Design Case," Biometrika, Vol. 3, 1988, pp. 591-602.

[5] J. P. Gould and C. R. Nelson, "The Stochastic Structure of the Velocity of Money," The American Economic Review, Vol. 64, No. 3, 1974, pp. 405-417.

[6] M. Friedman and A. J. Schwartz, "A Monetary History of the United States 1867-1960," Princeton University Press, Princeton, 1963. 